\title{
Modelling and simulation of a bioinspired aquatic micro aerial vehicle
}

\author{
S.F. Armanini; R.J.D. Siddall ${ }^{\dagger}$ M. Kovačc $\check{c}^{\ddagger}$ \\ Imperial College London, SW7 $2 A Z$ London, UK
}

\section{Introduction}

Thanks to their small size, low cost and relatively simple and fast manufacturing process, micro aerial vehicles (MAVs) facilitate the exploration of new and unconventional designs, which can allow for challenging tasks to be performed more efficiently or for new applications altogether. Especially for unconventional MAVs, a rich source of inspiration comes from nature. ${ }^{1-5}$ Bioinspired approaches can lead to MAVs with remarkable novel capabilities. These may directly emulate their biological counterpart, as in the case of many flapping-wing MAVs, but may also select only specific aspects of biological flight/behaviours to provide specific additional capabilities without incurring the considerable complication of a fully bioinspired design, ${ }^{6}$ which may not be optimal for the robotic case.

One desirable capability observed in biological flyers and currently unmatched by man-made vehicles, is the ability to transition autonomously from flight to water and vice versa, as for instance seen in diving birds . 7,8 Endowing MAVs with this capability - i.e. combining the advantageous features of flight and underwater operation - would allow for many new applications, such as water sampling (e.g. to monitor disaster scenarios or perform scientific measurements), or inspection of underwater infrastructure. Such tasks often require access to unstructured and potentially hazardous outdoor environments, and can be challenging to perform. At present, such applications typically involve a combination of different vehicles (e.g. water vessels carrying submarines) $)^{9}$ - an often costly and inefficient approach, due to the added complexity of integrating different vehicles in the same mission. Using aquatic MAVs for such tasks instead could considerably reduce the required resources, manpower, and safety risks, resulting in a higher overall efficiency, by allowing for single multi-purpose vehicles to be deployed where needed. However, operating in both water and air, and especially transitioning between the two, represents a considerable challenge and design tradeoff. Hence, while significant research on such vehicles has appeared in the past few years, ${ }^{5,9}$ the development of fully functional and automated vehicles capable of multiple transitions still represents a considerable active area of research.

Unsurprisingly, given the relative novelty of this field (particularly in terms of unmanned, small-scale vehicles), the vast majority of research on aquatic MAVs to date has focused on addressing the fundamental design and propulsion challenges. Several prototypes of such hybrid vehicles have been developed ${ }^{5,10-17}$ - many of these demonstrate single design concepts or partial aerial-aquatic capabilities, with only some demonstrating the full mission spectrum on a prototype. ${ }^{14-16}$ Nonetheless, even the latter do not yet demonstrate a fully functional, controlled and automated vehicle capable of efficiently performing full missions. Building on the considerable design research available, the next step towards the complete realisation of such vehicles is to improve the control and navigation aspect, allowing for automated, safe and eventually autonomous operation. To support this process, a comprehensive understanding of the dynamics of such MAVs is required. Dynamic modelling studies are still relatively scarce and typically focused on rotary-wing MAVs. ${ }^{18}$ Particularly more unconventional designs have not yet been the subject of extensive modelling work.

In this paper we develop a simple multi-phase model of the dynamics of a bird-inspired aquatic MAV (AquaMAV), with the aim of supporting the development of navigation and control strategies, facilitating

\footnotetext{
*Research Associate, Aerial Robotics Laboratory, Department of Aeronautics, Imperial College London. AIAA Member.

${ }^{\dagger}$ Research Associate, Aerial Robotics Laboratory, Department of Aeronautics, Imperial College London.

${ }^{\ddagger}$ Director, Aerial Robotics Laboratory, Department of Aeronautics, Imperial College London.
} 
the investigation of minor design changes, and providing further insight into the dynamics of bioinspired aquatic MAVs. The simulated vehicle is based on prior research on propulsion and design approaches for multi-modal locomotion ${ }^{5,11}$ and exploits a combination of variable wing-folding and conventional actuation and propulsion mechanisms to operate effectively in water and air, as well as transition effectively between the two domains. The mechanical design of this type of vehicle has been studied in detail in previous research by Siddall et al., 5,11 where initial models have been developed to predict dive trajectories. The current work extends and integrates previous research to construct a complete dynamics model that includes all operational phases and, especially, detailed consideration of the air-to-water and water-to-air transition phases including time-varying effects.

The devised dynamics model comprises three basic components, representing, respectively, the aerial, underwater and transition phases of operation. The aerial component, and part of the underwater component, are derived from wind tunnel data collected on a prior prototype of the platform, using a system identification approach. This results in a realistic representation of a significant part of the system. The remainder of the model, and particularly the transition phase, is based on simplified analytical approaches. The resulting model provides a detailed representation of the MAV, while also retaining a relatively simple structure, amenable for practical applications. The model is used to simulate and analyse the dynamics of the (type of) vehicle studied in all phases of operation. By providing both insight and a simulation framework, the developed model constitutes a useful basis for advanced control and navigation work on the unconventional type of vehicle studied.

\section{Vehicle geometry and preliminary considerations}

The model presented in this paper is for an unconventional type of MAV design. While the main aim was to conduct a generic analysis applicable to different forms of the same type of vehicle, that can be helpful when designing a specific type of platform, and therefore the details of the platform are not of primary importance, in order to obtain quantitative results, a specific design was used as a baseline, i.e. the AquaMAV developed by Siddall et al. ${ }^{5,10,11,19}$ In particular, the geometric properties of the simulated vehicle are largely based on the prototype presented in Ref. 11, and the aerodynamic model is derived from the wind tunnel data discussed in the same reference.

The main differences to the baseline vehicle are: (1) inclusion of conventional control surfaces on the tailplane, (2) conventional T-tail instead of V-tail, (3) absence of the water thrust mechanism for water exit, (4) consideration of an epicyclic gearing mechanism, ${ }^{19}$ allowing for effective propulsion in both air and water. (1) was deemed necessary in order to allow for later control and navigation work, the main intended application of the devised model. (2) was introduced to facilitate the actuation by decoupling the longitudinal and lateral control. (3) was introduced because the ejection approach is still the subject of further studies and hence excluding the water thruster makes the simulation both simpler and more general. With applications in mind, it is considered simpler to use conventional propellers to extract the vehicle from the water.

The simulated vehicle is assumed to have completely foldable elliptic wings, a wing span of approximately $0.6 \mathrm{~m}$ and a mass of approximately $0.2 \mathrm{~kg}$. It can be controlled by means of the wing folding $\left(0-90^{\circ}\right)$, through conventional elevator and rudder surfaces in the tailplane, and through thrust (throttle) variations. The use of ailerons for additional lateral control will be considered in future work - it may be possible to rely on the wing folding alone, thus reducing the number of actuators, however this would come at the price of a higher complexity in the roll mechanism. The general design mechanism is described in more detail in Ref. 11, while the key features are also provided in Table 1 below, for the limit cases of completely open (no sweep) and completely closed wings (90 degree sweep).

The model developed in the subsequent section is expressed in a body-fixed coordinate system, centred at the instantaneous CG of the body and aligned with the body axis, as clarified by Fig. 2(a). Note that when the wings fold, several changes occur in the vehicle configuration/ geometry, i.e. the wing area, wing chord and wing span change. Furthermore, the CG shifts backwards, and hence the inertia moments change. Simple linear functions were used to approximate these changes.

The body is assumed to be symmetrical around the longitudinal $\left(x_{B}\right)$ axis, and the wings move symmetrically and only in the $x y$ plane. Hence the combined CG of the two wings only moves in $x$-direction, as the changes in $y$ direction cancel out between the left and right wing. Using the new wing CG, the total CG of the body can be computed, and from it the moments of inertia. 


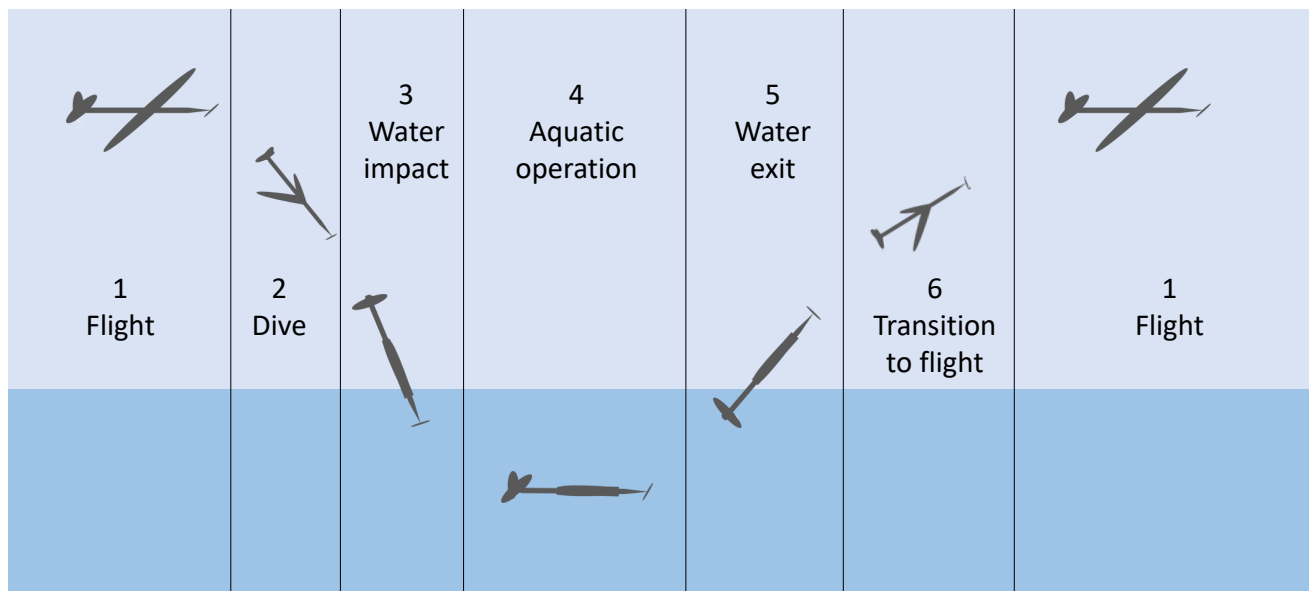

Figure 1. AquaMAV: envisaged multi-modal MAV mission, involving (1) flight, (2) plunge-dive manoeuvre, (3) water impact, (4) underwater operation, (5) water exit, (6) transition to flight and (1) flight again

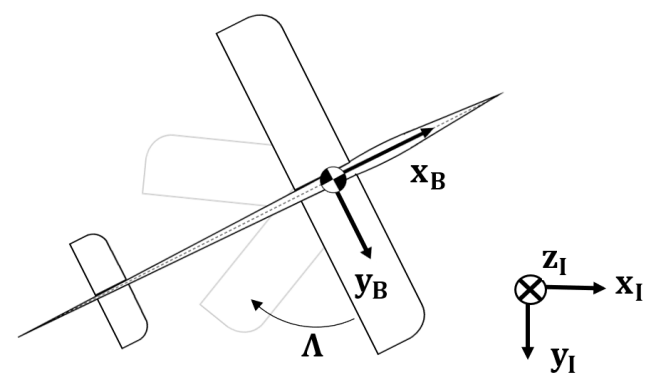

(a) Schematic of the vehicle (top view), showing body-fixed coordinate frame, inertial coordinate frame and definition of the sweep angle $\Lambda$
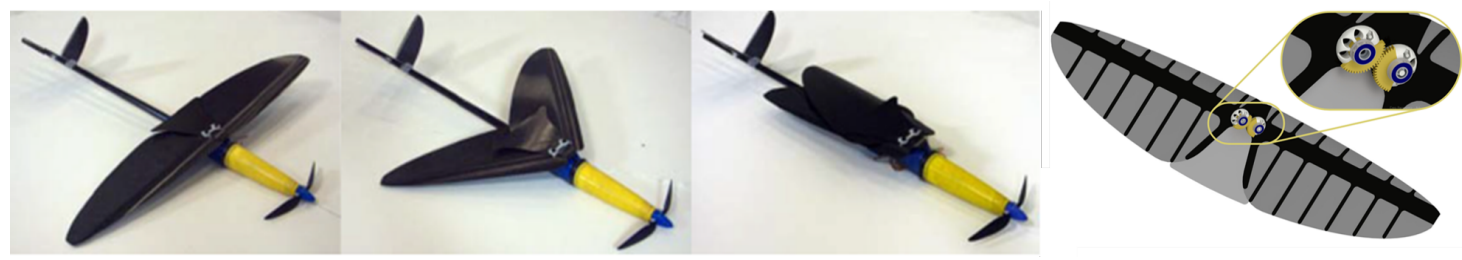

(b) Similar prototype vehicle: detail of folding wings (figure adapted from Ref. 11)

Figure 2. Prototype vehicle used as baseline for simulation, upon which many of the modelling assumptions were based. ${ }^{11}$

Table 1. Main features of the simulated MAV.

\begin{tabular}{llll}
\hline Property & \multicolumn{3}{c}{ Value } \\
& (open wings) & & (closed wings) \\
\hline$m[k g]$ & & 0.2 & \\
$x_{C G}[m]$ (from nose) & -0.19 & -0.21 \\
$I_{y y}\left[\mathrm{Nm}^{2}\right]$ & 0.0038 & 0.0043 \\
\hline Wing span $[\mathrm{m}]$ & 0.59 & 0.15 \\
Wing surface $\left[\mathrm{m}^{2}\right]$ & 0.06 & 0.02 \\
Horiz. tail span $[\mathrm{m}]$ & & 0.19 & \\
Horiz. tail root chord $[\mathrm{m}]$ & & 0.05 & \\
Horiz. tail surface $\left[\mathrm{m}^{2}\right]$ & & 0.01 & \\
\hline
\end{tabular}


Note that while several assumptions were made in the modelling process, the main goal of this study was not to develop a highly accurate model of a particular platform but to construct a somewhat flexible model of the type of MAV design. The obtained simulation framework will allow for control and navigation approaches to be designed and tested, and, when used in practice, the model can be adapted to the actual design studied.

\section{Modelling}

The overall model is comprised by several components, which are discussed further in the rest of this section. To narrow down the problem, the focus is placed on the longitudinal dynamics and forces. Many of the key functionalities and features can be analysed to a large extent in a vertical plane, before adding the complexity of the lateral component. Furthermore, the lateral aerodynamic forces were not characterised in the wind tunnel. Given the extensive range of sweep angles considered, existing studies do not provide a complete and reliable overview of the behaviour of the vehicle throughout this range.

Based on previous work on this type of design,${ }^{11}$ the equations of motion (EOM) for the longitudinal dynamics were defined in a conventional way, with additional terms to cover the impact and underwater phases.

$$
\begin{aligned}
\dot{q} & =\frac{1}{I_{y y}}\left[M-F_{B}\left(x_{\text {bary }}-x_{C G}\right) \cos \theta+T z_{C G}\right] \\
\dot{u} & =\frac{X}{m}-q w-g \sin \theta+\frac{F_{B} \sin \theta}{m}-\frac{F_{A M, u}}{m}+\frac{T}{m} \\
\dot{w} & =\frac{Z}{m}+q u+g \cos \phi \cos \theta-\frac{\cos \theta F_{B}}{m}-\frac{F_{A M, w}}{m} \\
\dot{\theta} & =q
\end{aligned}
$$

where $u$ and $w$ are the translational velocities along the $x_{B}$ and $z_{B}$ axes; $q$ is the body pitch rate, $\Theta$ is the pitch attitude, $m$ is the body mass; $I_{y y}$ is the body moment of inertia about the $y$ axis; $X$ and $Z$ are the aerodynamic forces in $x_{B}$ and $z_{B}$ direction; $M$ is the aerodynamic moment about the $y_{B}$ axis; $F_{B}$ is the buoyancy force; $g$ is the acceleration due to gravity; $T$ is the thrust force; $F_{A M}$ are the added mass force terms; $T$ is the thrust force from the propeller; and $x_{C G}$ and $y_{C G}$ describe the position of the CG along the respective body-frame axes, with respect to the vehicle nose. The forces in the above equations are discussed in the remainder of this section, with emphasis on the unconventional components arising from the unconventional design of the MAV studied, such as $0^{\circ}$ to $90^{\circ}$ folding wings and air-water transitioning.

\section{A. Aerodynamic forces and flight phase}

In view of the envisaged application of the aerodynamic model for simulation and control work, a smooth global model was developed to represent the vehicle aerodynamics. A system identification process was used for this, based on wind tunnel force measurements obtained on a similar type of platform ${ }^{11}$ (cf. Sec. II). Using experimental data to represent the aerodynamics, as opposed to analytical approximations, allowed us to model the effects of the extreme wing sweep changes in a realistic way, as well as to consider possible side effects related to the mechanical design, e.g. the wing-folding mechanism, which involves parts of the wings sliding beneath each other. ${ }^{11}$ While such effects are platform specific, and may vary significantly even between different realisations of the same platform, they are representative of the typical issues that may arise in comparable vehicles. As such, it can be useful, at least on a qualitative plane, to model these effects.

In the aforementioned wind tunnel tests, the aerodynamic coefficients of the separate components of the MAV (body, tail, wings) were measured at different angles of attack (AOA) $\left(-2^{\circ}\right.$ to $14^{\circ}$ in intervals of $\left.2^{\circ}\right)$ and, where relevant, different wing sweep angles $\left(0^{\circ}\right.$ to $90^{\circ}$ in intervals of $\left.10^{\circ}\right) .{ }^{11}$ The aerodynamic coefficients for the longitudinal forces and moments were thus assumed to depend on the AOA and wing sweep angle only. Reducing the aerodynamics to the most dominant effects was considered sufficient for a first approximation, although considering additional effects, e.g. angular rate effects, may prove necessary if rapid manoeuvres are considered. Thanks to separate measurements performed on the different vehicle components, each component could be modelled separately, which is helpful when modelling the water-air transition phases (cf. Sec. C), where different parts of the body enter/exit the water at different instants. 
Except for the large-range wing-folding, the MAV during flight is a conventional fixed-wing MAV. The body and tail coefficients were thus found to be accurately approximated by simple second-order functions of the AOA, with coefficients obtained via linear regression. Note that, as mentioned in Sec. II, the wind tunnel data was obtained for a $\mathrm{V}$-tail whereas the simulated vehicle was assumed to have a conventional T-tail: it was assumed, as a first approximation, that the simulated tail would generate the same overall aerodynamic forces as the $\mathrm{V}$-tail characterised in the wind tunnel.

By contrast, the wing coefficients were found to vary in a more complex way, due to the large range of sweep angles considered. While several aircraft designs include sweeping wings, the variation in sweep is typically confined to a smaller range. It is also typical for these vehicles to only operate at two or three discrete sweep configurations, rather than exploiting the full range. In this sense, the folding mechanism in the AquaMAV is unconventional, both due to the covered range and due to the possible utilisation of this range. While the final design may eventually involve switching only between a limited number of sweep angles, a full use of the available sweep range may also be considered. Hence, the model was required to provide full and continuous coverage of the sweep range.

The wing aerodynamic coefficients were found to depend nonlinearly on the AOA and the sweep angle. The nonlinearity in the wind tunnel data may also have been accentuated by the folding mechanism. While it is difficult to distinguish between aerodynamic and mechanical effects/ disturbances, it was considered of interest to capture the most evident irregularities in the model, as discussed above.

To obtain a flexible and smooth global model without resorting to high-order model structures, we used a model identification approach based on multivariate simplex B-splines. This approach allows for accurate models of highly complex datasets to be obtained by combining low-order local models. The underlying idea is to divide the identification domain into a net of non-overlapping simplices (triangulation), identify a local model for each simplex using the datapoints contained within it, and enforce continuity conditions of the desired order between neighbouring simplices. In particular, each simplex $t_{j}$ in the triangulation has a local barycentric coordinate system and supports a local polynomial of the form:

$$
p(x)=\left\{\begin{array}{rll}
\sum_{|\kappa|=d} c_{\kappa}^{t_{j}} B_{\kappa}^{d}\left(b_{t_{j}}(x)\right) & \text { for } & x \in t_{j}, \\
0 & \text { for } & x \notin t_{j}
\end{array}\right.
$$

where $c_{\kappa}^{t_{j}}$ are the coefficients of the polynomial (B-coefficients), $b_{t_{j}}$ are the barycentric coordinates of point $x$ with respect to simplex $t_{j}, d$ is the degree of the polynomial, $\kappa=\left(\kappa_{0}, \kappa_{1}, \ldots, \kappa_{n}\right)$ is a multi-index containing all permutations that sum up to $d$, and $B_{\kappa}^{d}(b)$ are the local basis functions of the multivariate spline:

$$
B_{\kappa}^{d}(b):=\frac{d !}{\kappa !} b^{\kappa}, \quad d \geq 0, \kappa \in \mathbb{N}^{n+1}, b \geq 0
$$

In a system identification context, the model structure thus locally takes the form of Eq. 5, with the Bcoefficients representing the model parameters and the basis functions being the independent variables used to model the system output. Standard estimators can then be used to estimate the parameters. An in-depth discussion of this identification approach can be found in Ref. ${ }^{20}$

The outlined approach was applied, with an ordinary least squares estimator, to obtain a model for the wing lift and drag coefficients, respectively, as a function of the wing sweep and AOA. The model structure and triangulation used for each model were based on an evaluation of the wind tunnel data, and fine-tuned in order to obtain an accurate and plausible result using the lowest possible model degree and smallest number of coefficients. Given that the model was intended to be used for control and generally within optimisation schemes, at least first order continuity was considered necessary. The chosen model structures and their accuracy are presented in Table 2, while Figs. 3 and 4 show the obtained models and original datapoints it can be seen that a high accuracy is achieved and a high proportion of the data are captured, providing a realistic basis for the current simulation.

In summary, the aerodynamic coefficients of the MAV take the following form:

$$
\begin{aligned}
C_{L, w} & =f^{3}(\alpha, \Lambda), & C_{D, w} & =f^{3}(\alpha, \Lambda) \\
C_{L, t} & =f^{2}\left(\alpha_{t}\right), & C_{D, t} & =f^{2}\left(\alpha_{t}\right) \\
C_{L, b} & =f^{2}(\alpha), & C_{D, b} & =f^{2}(\alpha)
\end{aligned}
$$


where the subscripts $w, t$ and $b$ denote, respectively, the wings, the tail and the body. The forces for each component $i$ were then computed according to the standard quasi-steady formulation:

$$
\begin{aligned}
L_{i} & =\frac{1}{2} \rho S_{i} C_{L, i} V_{\infty, i}^{2} \\
D_{i} & =\frac{1}{2} \rho S_{i} C_{D, i} V_{\infty, i}^{2}
\end{aligned}
$$

where $L$ is the lift, $D$ is the drag, $\rho$ is the fluid density, $S_{i}$ is the relevant surface area, and $V_{\infty}$ is the free-stream velocity experienced by the considered component. Note that when computing the tail forces, the velocity and $\mathrm{AOA}$ in the above expressions consider the effect of the body pitch rates, i.e.:

$$
\begin{aligned}
V_{t} & =\sqrt{u_{t}^{2}+w_{t}^{2}} \\
\alpha_{t} & =\arctan w_{t} / u_{t} \\
\text { with } \quad w_{t} & =w+q\left(x_{t}-x_{C G}\right), \quad u_{t}=u_{b}
\end{aligned}
$$

where $u$ and $w$ are the velocities in $x_{B}$ and $z_{B}$ direction, respectively, $x_{t}$ is the tail position along the $x_{B}$ axis and the subscript $t$ stands for tail.

The moments due to the wings and tail are then given by:

$$
\begin{aligned}
M_{t} & =\left(x_{C P, t}-x_{C G}\right) Z_{t} \\
M_{w} & =\left(x_{C P, w}-x_{C G}\right) Z_{w}
\end{aligned}
$$

where the force application points, indicated by the subscript $C P$ (centre of pressure), were likewise modelled from the wind tunnel data as simple functions of the AOA (and sweep angle). The tail force in the above expression includes the effect of potential elevator deflections.

Table 2. Modelling parameters and resulting model performance for wing aerodynamic coefficients.

\begin{tabular}{cccccccc}
\hline \multirow{2}{*}{ Output } & \multicolumn{3}{c}{ Model definition } & & \multicolumn{3}{c}{ Model performance } \\
\cline { 2 - 4 } \cline { 6 - 8 } & degree & continuity & \# simplices & & RMS & RMS [\%] & $R^{2}$ \\
\hline$C_{L}$ & 3 & 2 & 8 & & 0.06 & 2.4 & 0.99 \\
$C_{D}$ & 3 & 2 & 8 & 0.02 & 3.8 & 0.99 \\
\hline
\end{tabular}
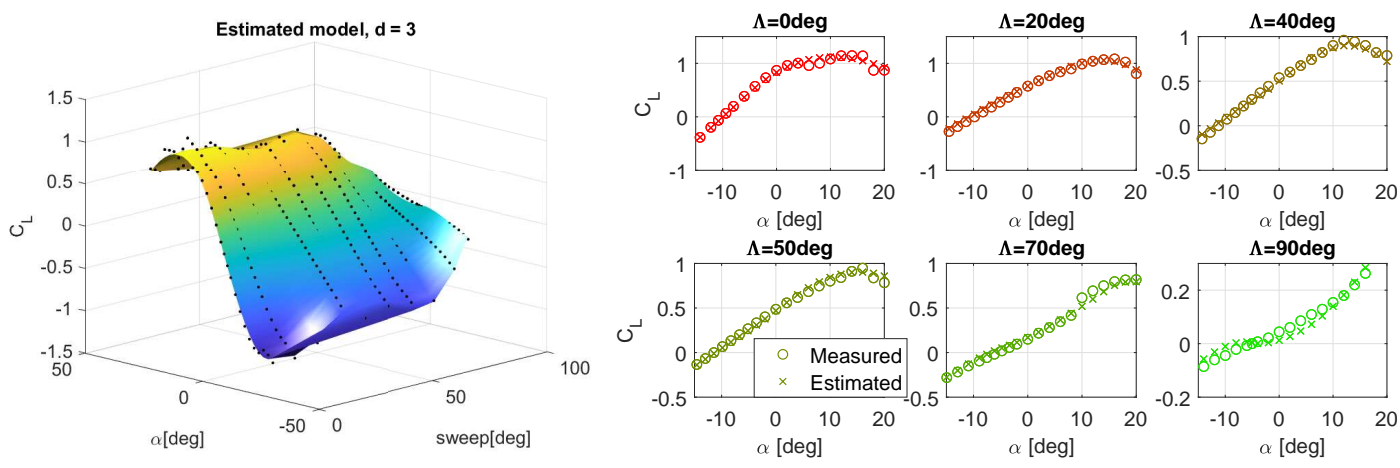

(a) Measured wind tunnel data (dots) and (b) Wind tunnel data versus model, for different sweep anmodel (surface) gles $\Lambda$

Figure 3. 3rd order spline model for $C_{L, \text { wing }}$

In addition to the basic lifting surfaces, the vehicle was assumed to have conventional control surfaces, as well as a propeller. The elevator effectiveness was estimated based on the assumed vehicle and tail geometry, ${ }^{21}$ and refined qualitatively considering the output to standard input signals - as for example shown in Fig. 6(b), which presents the response to a small elevator doublet. The propeller was approximated in a simplified way, based on the maximum static thrust predicted by the analysis in Ref. 19. 

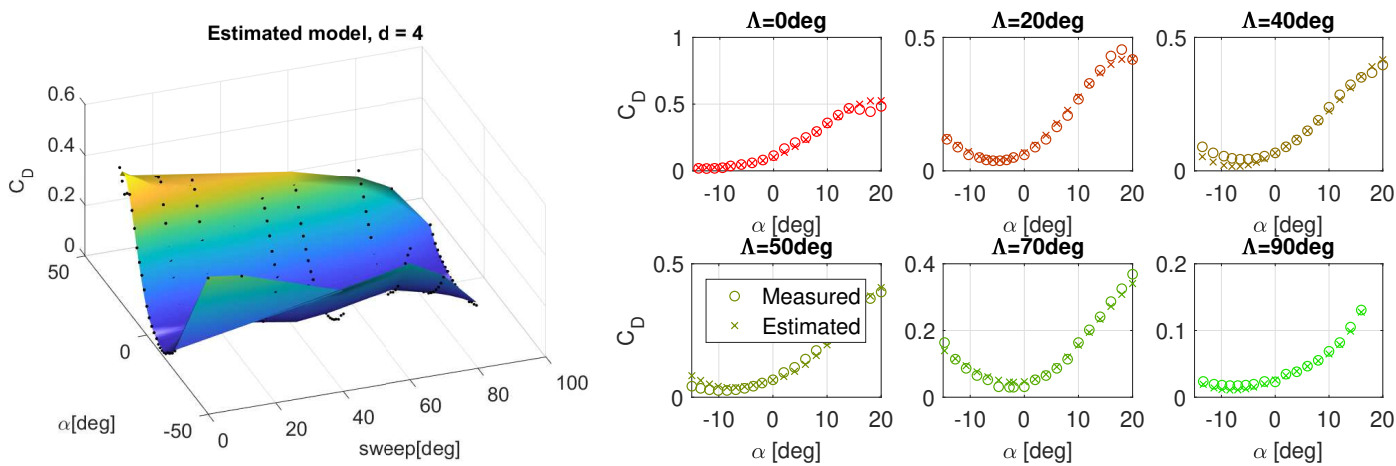

(a) Measured wind tunnel data (dots) and (b) Wind tunnel data versus model, for different sweep anmodel (surface) gles $\Lambda$

Figure 4. 3rd order spline model for $C_{D \text {,wing }}$

\section{B. Underwater phase}

In the underwater phase, the vehicle dynamics are determined by hydrodynamic, static and added mass forces, as well as propulsive and actuating forces introduced by the propeller and control surfaces. Based on the envisaged vehicle design, the wings were assumed to remain folded at all times while underwater.

The hydrodynamic forces were considered to be analogous to the aerodynamic forces, with the density in Eqs. $10-11$ being replaced by water density $\left(\rho_{w}=997 \mathrm{~kg} / \mathrm{m}^{3}\right)$ rather than air density $\left(\rho_{a}=1.225 \mathrm{~kg} / \mathrm{m}^{3}\right)$.

The buoyancy forces depend on the MAV volume. As for the reference test platform, a positive buoyancy was desired for the simulated vehicle - such that the vehicle will return to the surface automatically for water exit, as well as in case of a failure during underwater operation. As a simplification, the wings and tail were neglected in the volume calculation. The body geometry was approximated as a simple symmetric solid of revolution, with the cross section approximating the vehicle shape. In particular, as can be seen in Fig 2, the body is wider at the front, where the payload is located, while the hind part is essentially a thin rod.

Lastly, underwater operation may involve non-negligible added mass forces. In aerial operation, added mass effects are negligible owing to the very low density of air. Even underwater, added mass terms are often considered comparatively negligible - however, due to the high fluid density, this is not always the case, depending on the specific geometry and mode of operation of a vehicle. While studies on UAVs and small submarines often neglect the added mass component altogether, other studies have suggested that this component can be significant, particularly for bodies transitioning between different fluids. ${ }^{22}$ Given that the added mass is in any case non-negligible during water-air transitions, the term was also retained for the underwater component of the model.

Added mass forces are challenging to measure in isolation, as they typically occur in conjunction with other forces. Deriving accurate analytical expressions for these forces can likewise be challenging, particularly for objects with relatively complex geometries, which are typically characterised using either CFD or experimental methods. ${ }^{23,24}$ The alternative is simplification, i.e. using elementary geometries to represent a body. After impact, the AquaMAV resembles a slender ellipsoid combined with a thin plate. Even though the wings may contribute less to the added mass than the rest of the body, the interaction between the different body parts may be significant. However, this effect is also challenging to capture. It has been suggested that the added mass of bodies consisting of combined elementary shapes can be obtained by summing the added masses of each sub-component, but that that this approximation sometimes leads to significant errors ${ }^{25}$ and hence is not necessarily more reliable than other, simpler approaches.

In view of the above considerations, the equivalent ellipsoid approach was adopted to represent the added mass forces. As discussed in Ref. 26, the added mass of a slender body (length $>$ width) can be roughly approximated by the added mass of an ellipsoid with the same volume and the same length. The 
added mass forces are conventionally defined as,

$$
\begin{aligned}
F_{A M, x} & =\lambda_{11} \dot{u} \\
F_{A M, z} & =\lambda_{33} \dot{w} \\
M_{A M, y} & =\lambda_{55} \dot{q}
\end{aligned}
$$

where $\lambda_{i j}$ are the added mass coefficients, representing the added mass in direction $i$ due to an acceleration in direction $j$. In view of the assumed symmetry of the vehicle, cross-coupling terms (where $i \neq j$ ) are considered negligible. In an intuitive sense, the added mass can be viewed as the mass of fluid displaced in each direction. The added mass coefficients of the equivalent ellipsoid along each relevant axis can then be obtained according to following approximations: ${ }^{26}$

$$
\begin{aligned}
& \lambda_{11}:=m_{f} k_{11}, \quad \text { where } k_{11}=\frac{A}{2-A} \\
& \lambda_{33}:=m_{f} k_{33}, \quad \text { where } k_{33}=\frac{C}{2-C} \\
& \lambda_{55}:=I_{f} k_{55}, \quad \text { where } k_{55}=\frac{\left(a^{2}-c^{2}\right)^{2}(A-C)}{\left(2\left(c^{4}-a^{4}\right)+(C-A)\left(c^{2}+a^{2}\right)^{2}\right)}
\end{aligned}
$$

where $a$ and $b$ are, respectively, the long and short semi-axes of the ellipsoid longitudinal cross-section, $A$ and $C$ are parameters depending on the geometry of the ellipsoid (see Ref. 26 for details), and $m_{f}$ and $I_{f}$ are the total virtual mass and inertia. As the MAV body was assumed to be approximately rotationally symmetric, the minor semi-axes of the ellipsoid were assumed to be equal and both denoted by $b . m_{f}$ and $I_{f}$ are thus:

$$
\begin{aligned}
m_{f} & =\frac{4}{3} \rho \pi a b^{2} \\
I_{f} & =\frac{4}{15} \rho \pi a b^{2}\left(a^{2}+b^{2}\right)
\end{aligned}
$$

\section{Air-water transitions}

The air-water and water-air transitions constitute the most challenging phase to model, representing highly complex multi-phase flow problems. During water entry, the violent impact at high velocities further adds to the complexity: as the body impacts the considerably more dense water, the flow is heavily disrupted and huge forces act on the body. As the two problems can be formulated in a similar way, we discuss the water entry case here and only point out the main differences considered in the water exit case.

The impact phase sees considerably large forces acting in a time-varying way over an extremely short period of time. The moment the vehicle comes into contact with the water surface, the drag forces increase drastically and buoyancy and added mass forces are introduced into the system. Furthermore, the forces are influenced by the continual variation in submerged body mass, surface area and volume of the body, as it transitions from one phase to the other, and by the resulting variation in impact geometry. Moreover, while the MAV is approximately axissymmetrical, it does not have an ideal shape, as even in a folded arrangement the wings and tail do not fully conform to the streamlined shape of the rest of the vehicle.

In the literature, the fluid-dynamic problem of water entry has been widely studied since the initial formulation by Wagner. ${ }^{27}$ Even now, many studies are based on assumptions, e.g. vertical impact, ideal symmetric shape, ideal fluid at rest, etc. ${ }^{22,28,29}$ While several more elaborate analytical formulations have been introduced, ${ }^{30}$ e.g. accounting for additional effects such as cavitation, or for complex geometries or kinematics, especially applied studies typically use CFD approaches or significant simplifications to simulate water entry. ${ }^{22}$ Given that the intended purpose of the current simulation model was to support the development of control systems and to provide a basic idea of the system dynamics, computationally heavy models, e.g. CFD-based ones, were not considered. Instead, the water entry was modelled in a highly simplified way. Nonetheless, an additional aim of the current study was to evaluate to what extent a highly simplified model is acceptable and how realistic the predicted outcomes are.

Thus, in addition to the assumptions introduced in Sec. B for the full submersion case, a number of additional assumptions were made specifically regarding the water entry process (and exit process, where relevant). In particular, while the impact was not constrained to the ideal vertical case, the impact surface 
was assumed to be analogous to the surface that would be obtained during a vertical impact - as clarified in Fig. 5. This allowed for the same simplified geometry to be assumed for the body throughout the impact phase, with the impacting surface approximated by a circular cross section (with varying width). Moreover, the instantaneous volume and surface in air/water could be more easily computed. Besides implicitly in the state and force components, the impact angle was considered in terms of the submerged body length, which was defined to be aligned with the body axis. All states were computed considering the impact angle.

The buoyancy force was computed as for the underwater case, with the instantaneous submerged volume replacing the volume of the full body. Similarly, the approximate barycentre position was computed considering the submerged part of the body.

The added mass forces were based on the same simplifications discussed in Sec. B, extended to the time-varying case. Comparable semi-empirical approaches have for instance been applied to missiles exiting water, ${ }^{22}$ and to conic wedges entering water ${ }^{29}$ - while the current study involves more extensive simplifications, this suggests that the type of approach is in principle applicable to water entry/exit problems. Unlike in the underwater case, the geometry of the body varies during the transition phases. Assuming the added mass in air is negligible, the total added mass can be assumed to depend only on the submerged part of the body. Hence, the added mass coefficients $\lambda_{i j}$ are no longer constant, but depend on the instantaneous geometry of the current equivalent ellipsoid. Furthermore, the submerged volume of the body changes during water entry/exit, influencing the virtual mass and inertia terms. Hence, the total added mass (Eqs. 16-18) was reformulated to include an additional term: ${ }^{22,29}$

$$
\begin{gathered}
F_{a m, x, \text { impact }}=\lambda_{11} \dot{u}+\dot{\lambda}_{11} u \\
F_{a m, z, \text { impact }}=\lambda_{33} \dot{w}+\dot{\lambda}_{33} w \\
M_{a m, y, \text { impact }}=\lambda_{55} \dot{q}+\dot{\lambda}_{55} q
\end{gathered}
$$

Recalling the previous definition of the added mass coefficients given in Eqs. 19-21, the derivatives $\dot{\lambda}_{i j}$ can be expressed as,

$$
\begin{array}{r}
\dot{\lambda}_{11}=\dot{k}_{11} m_{f}+\dot{m}_{f} k_{i i} \\
\dot{\lambda}_{33}=\dot{k}_{33} m_{f}+\dot{m}_{f} k_{33} \\
\dot{\lambda}_{55}=\dot{k}_{55} I_{f}+\dot{I}_{f} k_{55}
\end{array}
$$

where the virtual mass and inertia derivatives are given by

$$
\begin{aligned}
\dot{m}_{f} & =\frac{4}{3} \dot{L} \rho \pi b^{2} \\
\dot{I}_{f} & =\frac{4}{15} \pi \rho b^{2} \dot{L}\left(3 a^{2}+b^{2}\right)
\end{aligned}
$$

where $\dot{L}$ is the rate of change of the submerged length. By definition, in the current problem $\dot{L}=\dot{u}$, as the length is aligned with the body $x$-axis. The coefficient derivatives were obtained by analytical differentiation of Eqs. 19-21 (which, through the coefficients $A$ and $C$ depend on the instantaneous length submerged). Owing to the length of the obtained expressions, these are not reported here.

For the water entry case, the above formulation neglects potential cavitation or air entrainment effects. The influece of upsurge was also neglected based on preliminary tests which suggested the overall influence on the impact forces was relatively small. Note that the above approximations break down in the first instants after impact (and the final instants at water exit), as here the submerged geometry no longer resembles a slender body, but more closely represents a spheroid. To deal with this phase, the ratio $a / b$ was assumed to remain constant during these infinitesimal times - implying the submerged part of the body is approximated as a cone.

Finally, the drag (and potentially lift) forces were modelled. Two different approaches were considered. Firstly, it is possible, as a rough approximation, to assume - as in Sec. B for the completely submerged case - that the hydrodynamic forces take the same form as the aerodynamic forces in air, with only the density changing. Taking a similar approach as in Ref. 10, the submerged part of each component of the vehicle was determined based on the submersion geometry, and the force produced by each component was scaled by the submerged area. Thus, for example, the wing forces are given by,

$$
F_{w, \text { transition }}=\frac{1}{2} V^{2} C_{L, w i n g}\left(\rho_{w} S_{w}(t)+\rho_{a} S_{a}(t)\right)
$$




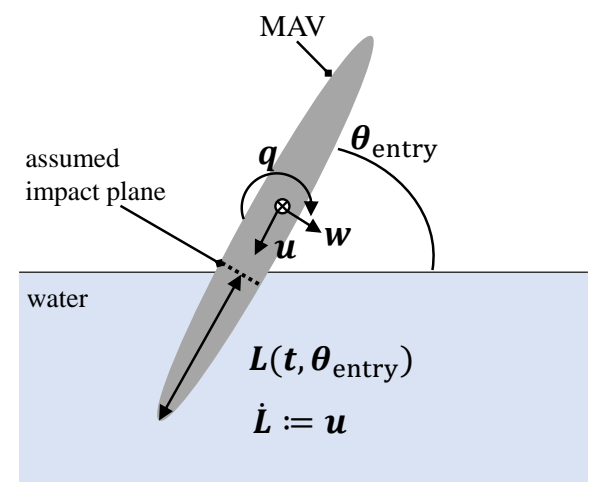

Figure 5. Impact geometry clarification. Note: the terminology is analogous for the water exit case, with the vehicle being reversed (such that velocity $u$ points upwards).

For the purpose of moment calculations, the force application points for each component were assumed to remain the same. The above expression may be acceptable for the water exit case. During the impact phase, however, the flow around the body is likely to be highly chaotic. Furthermore, cavitation or air entrainment may occur, which for instance may lead to the tail, and potentially parts of the wings, being ineffective during and shortly after the impact. In this sense, it was considered more realistic to assume that during and shortly after the impact, the hydrodynamic forces are dominated by skin friction effects, as also suggested in Ref. 10. Hence, in the alternative formulation we assume - as in Ref. 10 - that the drag force coefficient is given by:

$$
C_{D}=\frac{0.0307}{R e^{1 / 7}}
$$

where the Reynolds number is computed considering the submerged length of the body.

The above formulations provide an estimate of the forces occurring at water entry and exit, nonetheless they are highly simplified and may not be sufficiently realistic in practice. This will be discussed further when evaluating the results.

\section{Simulation results and system analysis}

The developed model allowed us to simulate and analyse the dynamics of the envisaged aquatic MAV in different phases of operation (cf. Fig. 1), deriving basic insight and requirements for control and effective overall operation. Particular emphasis was placed on the most relevant and unconventional manoeuvres, i.e. plunge-diving and air-water-air transitions. This section presents examples of simulation results, illustrating the full mission cycle and allowing for the plausibility of the model to be evaluated, followed by a brief appraisal of the system dynamics, with an outlook on control work. Finally, the transition phases are briefly considered in further detail.

\section{A. Simulation of different phases}

Figures 6-8 show a simulation of the different phases of operation of the modelled MAV, covering (i) steady and manoeuvring flight, (ii) steep dive manoeuvre, (iii) water impact and entry, (iv) steady underwater operation, (v) return to surface and water exit. The obtained results suggest that the devised model can effectively represent both the purely aerial aerial and aquatic phases, and the transitions from air to water and vice versa. The resulting system behaviour is physically plausible and agrees well with experimental results obtained previously on similar vehicles. ${ }^{11}$ It must be noted that, while all phases are covered, in order to achieve a continuous, full mission cycle, active stabilisation is needed for some parts of the cycle, e.g. stable water exit and transition back to flight, and transition from water impact to controlled underwater operation. The control component will be the subject of future work and is not discussed further here. 


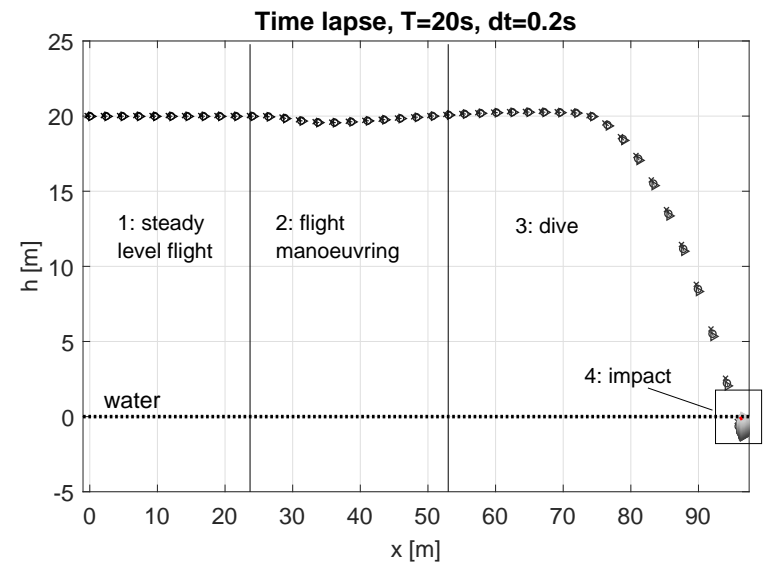

(a) Time lapse showing trajectory in longitudinal plane, body not to scale
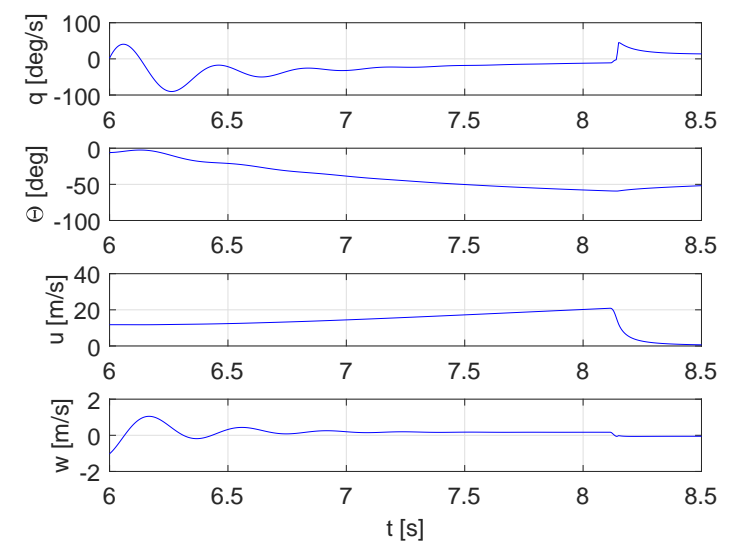

(c) State evolution, part 3: wing folding-initiated dive and water impact
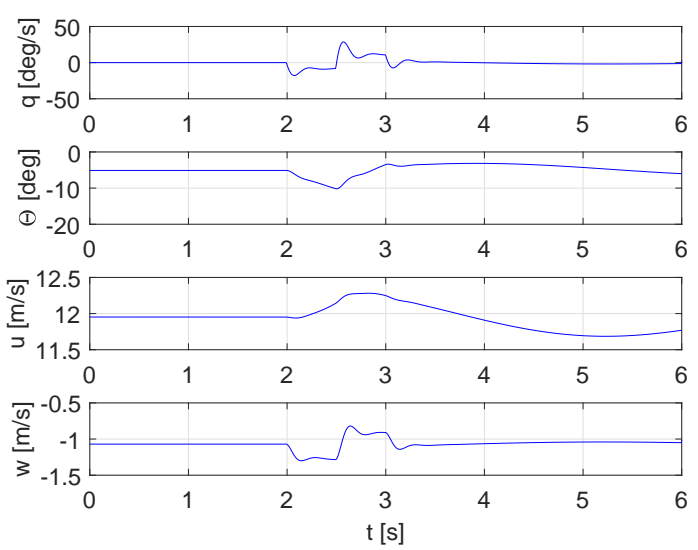

(b) State evolution, part 2: doublet manoeuvre in air
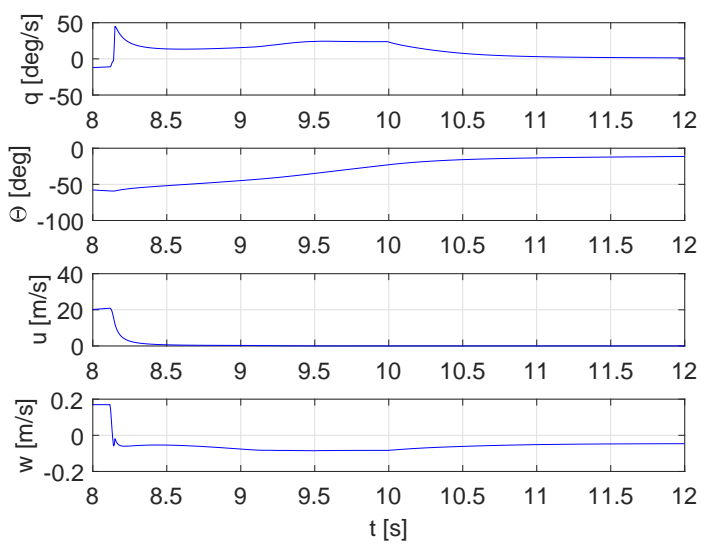

(d) State evolution, part 4: detail of water impact and transition

Figure 6. Simulation example: aerial phase and water impact.

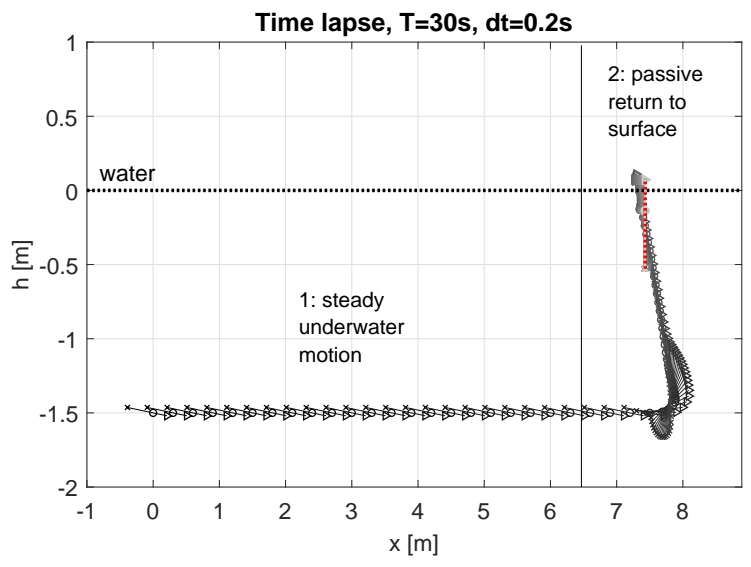

(a) Time lapse showing trajectory in longitudinal $(x z)$ plane, body not to scale
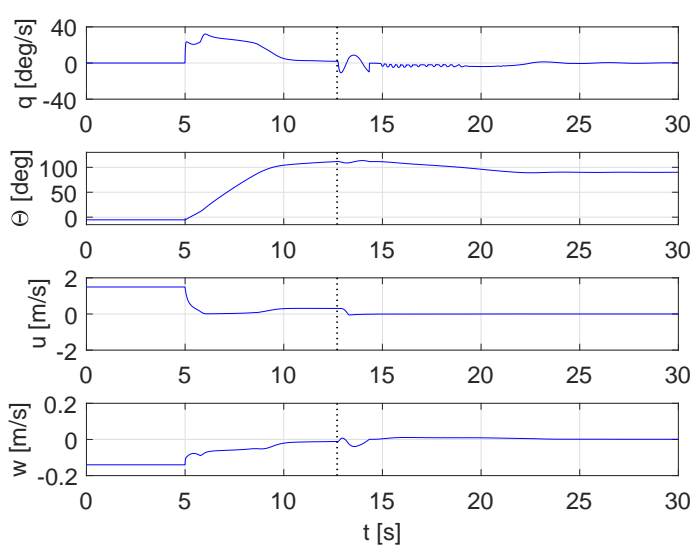

(b) State evolution

Figure 7. Simulation example: underwater phase and passive rise to surface. 


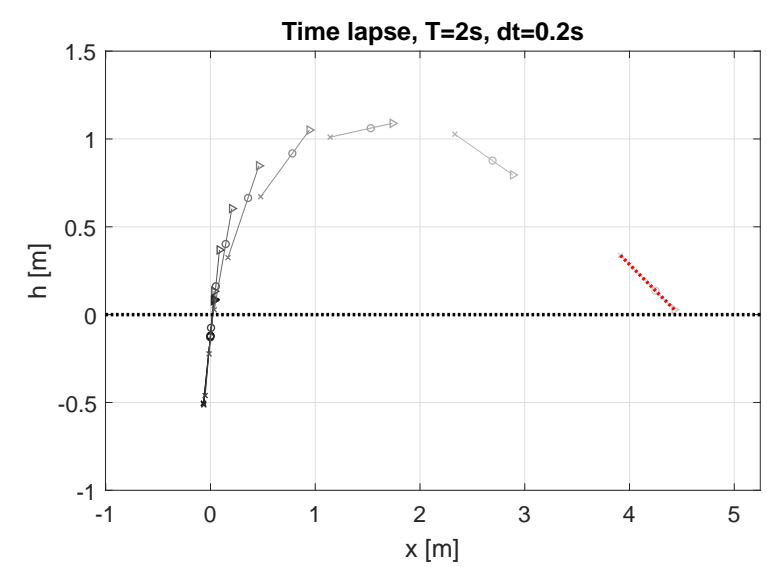

(a) Time lapse showing trajectory in longitudinal $(x z)$ plane, body not to scale
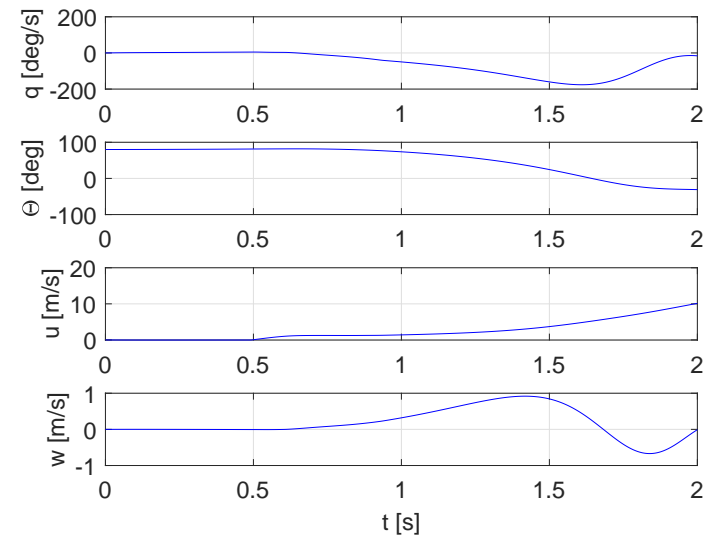

(b) State evolution

Figure 8. Simulation example: unstabilised launch from floating condition.

\section{B. System dynamics: aerial and aquatic operation}

To obtain more in-depth insight into the behaviour of the MAV, its operational envelope was determined and its dynamics were evaluated across the aforementioned envelope. Fig. 9 shows examples of numerically $\alpha-V$ trim curves obtained for the vehicle at a number of different wing sweep angles, in air and in water. In the interest of completeness, the underwater curve for open (unfolded) wings is also shown, depite the envisaged vehicle operating with closed wings while underwater. The obtained curves are qualitatively plausible, with a lower angle of attack being required to stabilise at higher speeds (note that the extensive negative angle of attack range is a result of the wing aerodynamics, cf. Sec. A). The typical operating speed in air and water was found to be, respectively, $\sim 10 \mathrm{~m} / \mathrm{s}$ and $\sim 1 \mathrm{~m} / \mathrm{s}$. The combination of wing sweep and elevator input leads to a fairly large envelope especially in flight. It is also interesting to note that the behaviour of the system is nonlinear with the sweep angle: changes are initially very gradual, then increasingly rapid, with the flight envelope changing rapidly between $\Lambda=60^{\circ}$ and $\Lambda=90^{\circ}$.

In the underwater phase the operational envelope is in a sense more restricted, as the wings remain folded. The envisaged epicyclic gearbox ${ }^{19}$ allows for adequate thrust production underwater: with the current setup, it was found to be possible to maintain the vehicle at a constant depth at velocities of 1.0$2.5 \mathrm{~m} / \mathrm{s}$. Holding a constant horizontal position, on the other hand, is virtually unachievable in practice due to the relatively limited underwater actuation possibilities. A neutrally buoyant alternative configuration, or some form of water surface vehicle would be better suited for missions requiring extended periods of time at an approximately constant position. Alternatively, more elaborate or additional underwater actuation mechanisms, e.g. a form of buoyancy control, may allow for more effective underwater manoeuvring and/or horizontal position-keeping. These options will be considered in future work.

To obtain further insight into the system dynamics, stability was evaluated at the trim points defining the stable operational envelope. This approach can be considered an acceptable first approximation for the steady aerial and subaqueous phases, though it cannot account for aggressive nonlinear manoeuvres. The nonlinear model (eqs. 1-4) was thus linearised around a set of steady conditions, based on the previously discussed trim results, and the resulting local linear models, of the form $\dot{\mathbf{x}}=\mathbf{A x}+\mathbf{B u}$ (with $\mathbf{x}:=[q, \theta, u, w]^{T}$, $\mathbf{u}=\left[\delta_{E}, \delta_{T}, \Lambda\right]^{T}$ ), were used for stability analysis of the aerial and aquatic phases. Stability during the transition phases, where the system becomes inherently time-varying, was not considered at this stage.

Fig. 10 shows the eigenvalues computed for a representative number of linearised models, representing different aerial and aquatic equilibrium conditions. As expected, the system dynamics are considerably influenced by the significant changes in configuration (wing-folding) and, especially, mode of operation (air/water). Clearly, one of the most significant challenges of the studied system is thus the combination of very different components, even if the single components may, on their own, be relatively uncomplicated. This also suggests that some form of control adaptation or switching may be required to allow for a fully-functional and autonomous vehicle. 


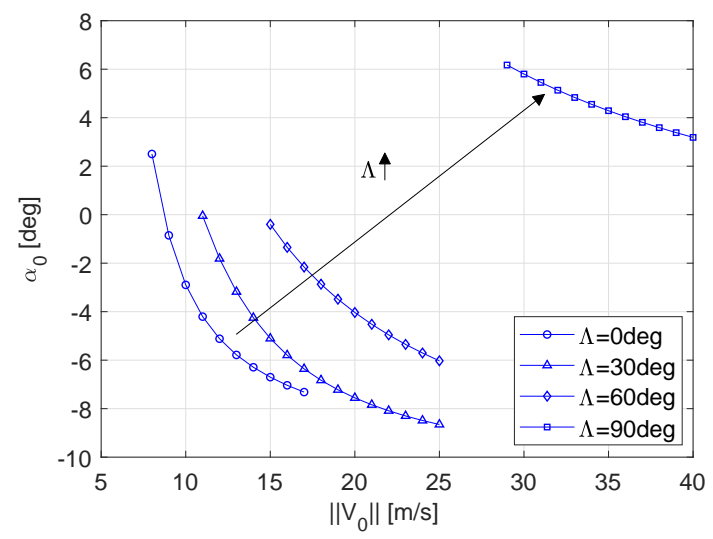

(a) Steady level flight trim curve.

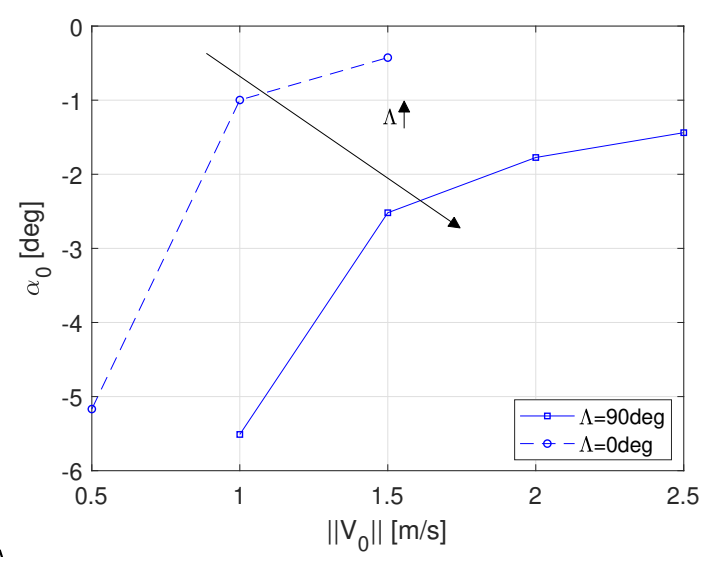

(b) Steady underwater operation trim curve.

Figure 9. Steady operational envelope in air and water, considering different wing sweeps.

In the aerial phase (Figs. 10(a)-10(b)), the system dynamics are conventional for the open-wings case, with two complex pole pairs that approximately correspond to short period and phugoid modes. With increasing sweep angle, the stability increases, with both pole pairs moving to the left in the complex plane. The slower mode additionally increases somewhat in damping, moving slowly towards the real axis with little change in frequency, while the faster mode increases significantly in frequency, with a small decrease in damping only at very high sweep angles. At low sweep angles, the slow mode is quite poorly damped. It is also interesting to observe that, echoing the trim curves, changes are gradual until sweep angles of approximately $60^{\circ}$ and then more rapid, presumably as a combined effect of the aerodynamics and the folding mechanism, which affects the wing profile. The underwater phase is shown in Figs. 10(c)-10(d) for the relevant foldedwings case. All eigenvalues were found to be aperiodic and stable. While the system is therefore stable, it must be borne in mind that the MAV is slightly positively buoyant and hence requires continual control inputs to remain underwater.

In addition to steady operation at constant altitude (or depth), an additional phase that is interesting to consider is the unpowered dive. Technically part of the aerial operation, this manoeuvre is nonetheless unconventional and constitutes a central element in the envisaged MAV's mission cycle as it enables the robot to enter the water. Fig. 11 shows the steady glide conditions achievable with different wing sweep angles. As already suggested in Ref. 11 for a predecessor robot, the wing-folding considerable affects the resulting trajectory and can potentially be used as sole mechanism to carry out a dive. Folding back the wings leads to terminal impact angles of up to $\sim 70^{\circ}$ - as the wings still generate lift when folded, the steady-state pitch attitude cannot be vertical. However, the additional use of conventional control surfaces potentially allows for larger impact angles to be attained, at slightly lower velocities. Given that steeper entry angles are generally preferred, as they allow for greater depth to be reached, reduce the potential damage of the impact, and lead to more predictable underwater trajectories, using conventional actuators in addition to the folding wings may be desirable. Nonetheless, the dive manoeuvre requires a degree of compromise. Achieving steep entry angles requires high velocities, which may be damaging to the MAV and furthermore lead to it covering large distances before reaching the desired attitude. During this time the robot is also less easily controlled due to the high speed and smaller lifting surfaces. In this sense, it is not advisable to reach terminal speeds, but rather to use a combination of actuator inputs to attain an acceptable non-steady entry condition.

\section{Outlook: analysis of transition phases}

The analysis in the previous section focused on the two main phases of operation of the studied MAV, however the main challenge is given by the far shorter transitions between these phases. Thanks to the full inclusion of time-varying effects, the developed model can be used to study the transition phases in some detail, also allowing for a full study of the stability, especially during the slower water exit manoeuvres. A detailed analysis of the transitions is left for future work, however a brief overview of initial simulation 


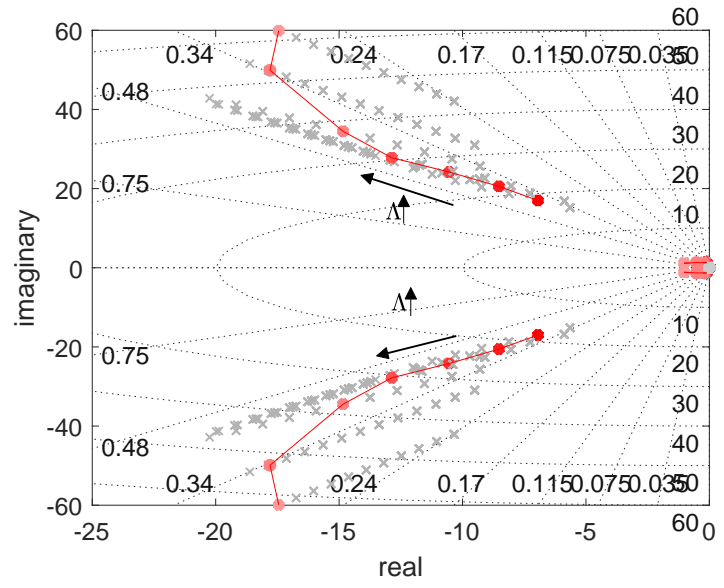

(a) Aerial operation, view highlighting fast mode

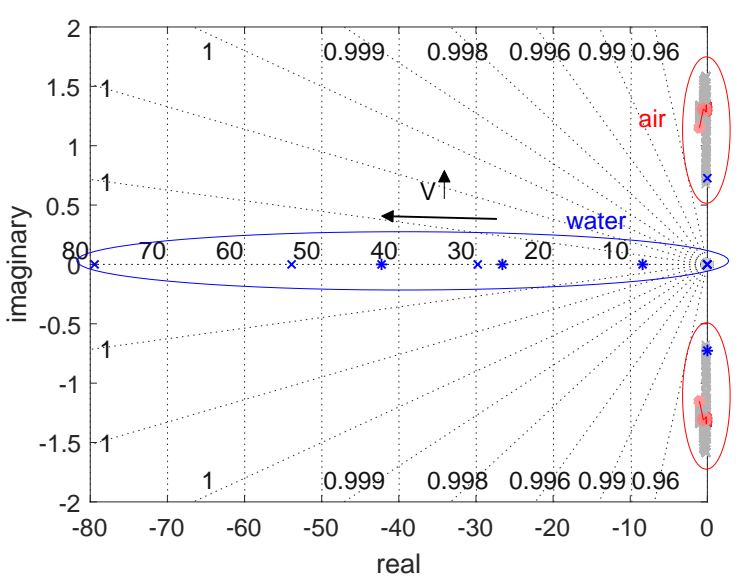

(c) View highlighting fast modes of underwater operation

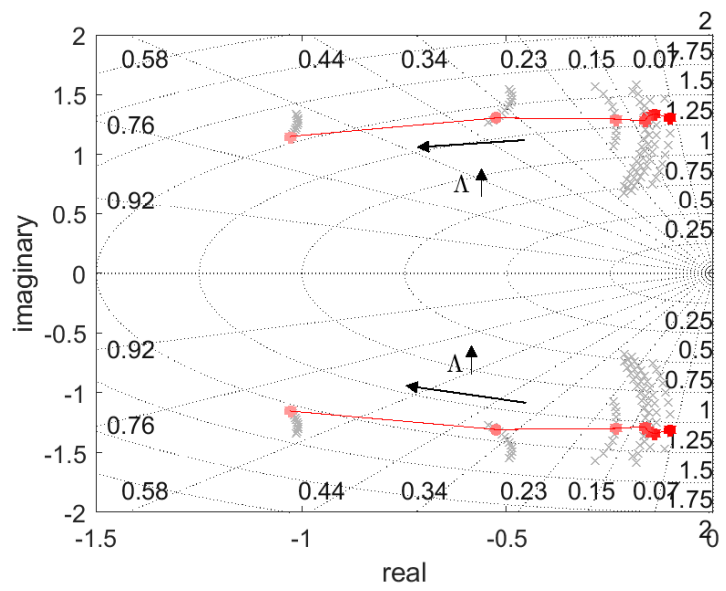

(b) Aerial operation, closer view highlighting slow mode

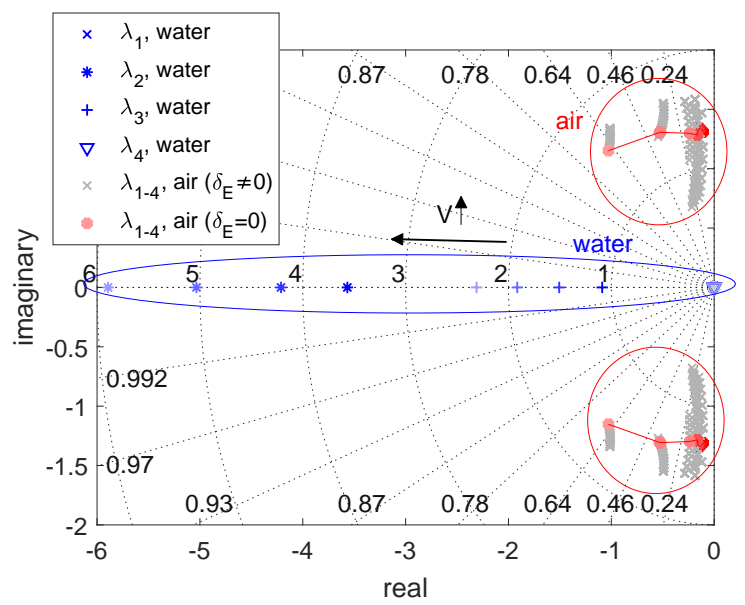

(d) View highlighting slower modes of underwater operation

Figure 10. Eigenvalues $\lambda_{i}$ of the system in aerial and underwater operation, in the vicinity of different steady operating conditions. For the aerial component, the red dots show the neutral-elevator case, while the grey crosses show the larger flight envelope achievable with additional controls. The underwater component is shown only for the case of closed wings and includes both throttle and elevator input.
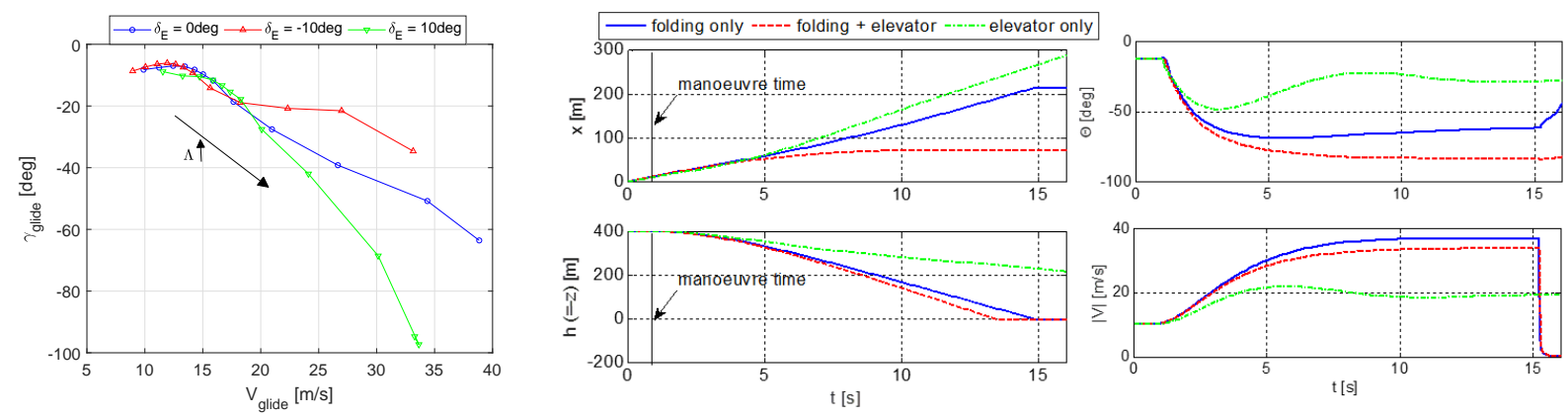

(a) Steady glide conditions for different (b) Example of large-scale dive manoeuvre using only wing-folding, only elevator sweep angles and elevator trim inputs input, or both.

Figure 11. Unpowered steady glide conditions and dive manoeuvre example, showing the glide angle $(\approx$ pitch attitude) and velocity magnitude attainable by changing the wing sweep only, or both the wing sweep and the elevator deflection angle. 
results is given in this section as an outlook.

Figs. 12-13 show the changes in the forces and states occurring during and immediately after water impact, for different entry angles and velocities. It can be observed that while the forces on the vehicle are huge, i.e. predicted to reach several hundred $g$, the time scale of the process is very short compared to the remainder of the system dynamics. This for instance suggests that small changes in the forces (e.g. due to slightly different impact model formulations) may have a relatively limited overall effect, especially on the resulting attitude and position. Moreover, given that the test conditions in any realistic scenario will be unideal and very difficult to predict or characterise, it is likely that the real system will deviate non-negligibly from the modelled system. In particular, the uncertainty deriving from external disturbances, as well as manufacturing inaccuracies, flexibility effects, etc., may be larger than the uncertainty in the model. Another consequence of the small time scale and very large changes occurring in the system is that even the current highly simplified representation of the transition phases requires a very small integration step for meaningful simulation results to be obtained, which adversely affects simulation speed and may limit online applicability. The combination of the above points suggests that aiming for an extremely high accuracy may not be necessary - instead, it is advisable to develop a controller that can handle a degree of model uncertainty and disturbance occurring during and immediately after the impact phase. For simulation purposes, a possible approach may be to represent the impact using a surrogate term acting only at a single instant, but approximating the time-varying forces of the entire impact process.

In terms of impact conditions, unsurprisingly, steeper impact angles and higher impact velocities allow for greater depths to be reached, with shallow angles and low velocities eventually leading to failure of the body to fully penetrate the water surface. The most noticeable effect of the initial impact velocity is on the depth and on the normal velocity component $w$ (Fig. 12), while the attitude obviously most influences the trajectory occurring after impact (Fig. 13). The predicted depths (cf. Fig. 13) attained are slightly higher than previous estimates that neglected unsteady effects, ${ }^{11}$ but remain in a comparable range.

The water exit phase, driven by a powerful propeller, is somewhat slower and less aggressive than the impact phase. Here the time-varying evolution of the forces is in a sense more important because it influences the feasible exit trajectories. While the exit phase will need to be further studied, with particular emphasis on the stability of the time-varying system, our model provides intial insight, allowing for the time-varying evolution of the forces and states to be estimated. An example of this is shown in Fig. 14, which presents the forces and positions obtained for a range of different exit angles, under the assumption that such angles can be achieved through underwater manoeuvring. As expected (and illustrated by Figs. 14(c)-14(e)), a more vertical exit angle tends to facilitate the exit process. Nonetheless, considering that the vehicle needs to transition back to horizontal flight once out of the water, it may be advantageous to exit the water at an angle. In this sense, it may be interesting, in future work, to consider different exit manoeuvre options, i.e. exiting from a near-vertical floating condition after passively rising to the surface, or commencing the exit manoeuvre underwater, hereby potentially exploiting some underwater velocity as well as buoyancy. An important point to consider in this evaluation is the effect of wetness on the efficiency of the propeller and the lifting surfaces, which at present was neglected.

Like the impact process, albeit to a lesser extent, the exit process is relatively fast and difficult to model accurately. Being less rapid and violent, it is however slightly more sensitive to inaccuracies. Furthermore, given that the exit process requires stabilisation, an accurate representation of the system is of greater relevance.

Nonetheless, despite significant differences in time scales and force magnitudes, the transitions in both directions share several characteristics. Considering that a high accuracy is challenging to achieve and the current model involves a number of simplifications, it is thus important, firstly, to attempt an experimental validation of the obtained results, and, secondly, to implement a robust control strategy for the exit process that can handle a degree of model uncertainty.

\section{Conclusions}

Aquatic micro aerial vehicles (MAVs), capable of transitioning repeatedly between aerial and underwater operation will allow for useful new applications, such as rapid autonomous water sampling. However, such vehicles are challenging to develop due to the requirement of operating in extremely different conditions, hence fully mission-capable and autonomous prototypes are not yet available, especially at small scales. In this paper, a model was constructed for a bird-inspired aquatic MAV, with the aim of fully characterising its 


$-\mathrm{V}_{\text {imp }}=35 \mathrm{~m} / \mathrm{s}$
$---\mathrm{V}_{\text {imp }}=25 \mathrm{~m} / \mathrm{s}$
$-\cdot--\mathrm{V}_{\text {imp }}=15 \mathrm{~m} / \mathrm{s}$
$---\mathrm{V}_{\mathrm{imp}}=5 \mathrm{~m} / \mathrm{s}$
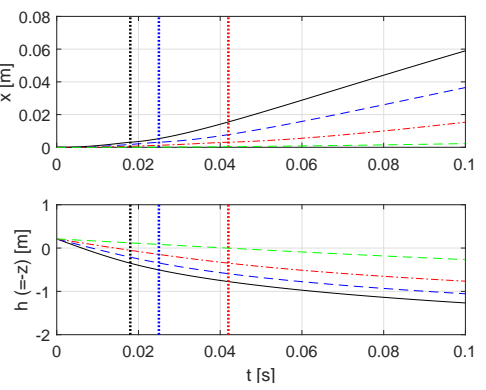

(c) Inertial-frame CG positions

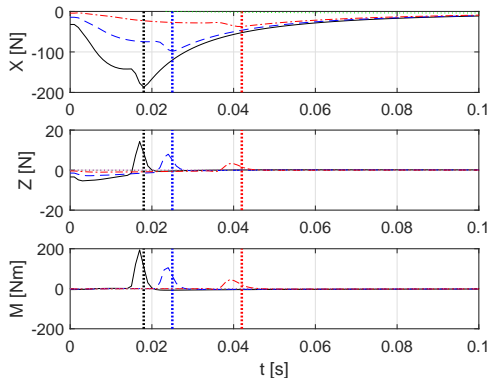

(a) Body-frame forces and pitch moment
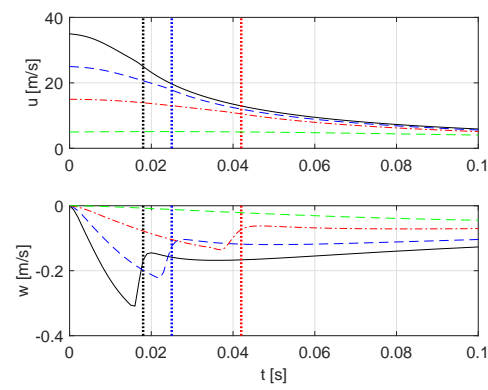

(d) Body-frame velocities
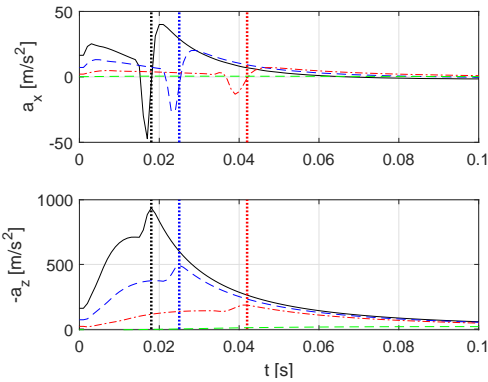

(b) Inertial-frame accelerations
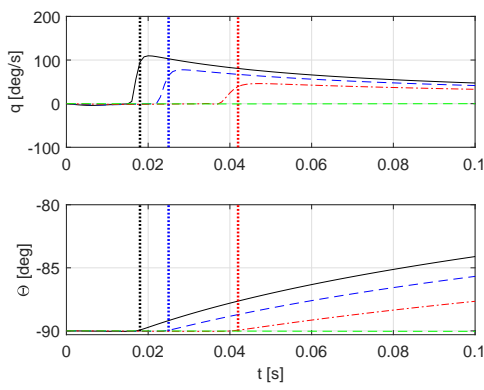

(e) Pitch rate and attitude

Figure 12. Water impact process for different body-aligned entry velocities $V_{i m p}$ (vertical entry angle); the dashed lines indicate the time at which the body is fully submerged.
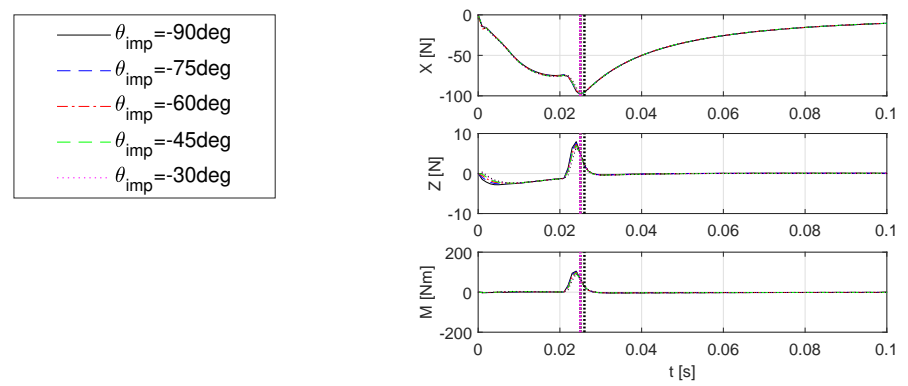

(a) Body-frame forces and pitch moment

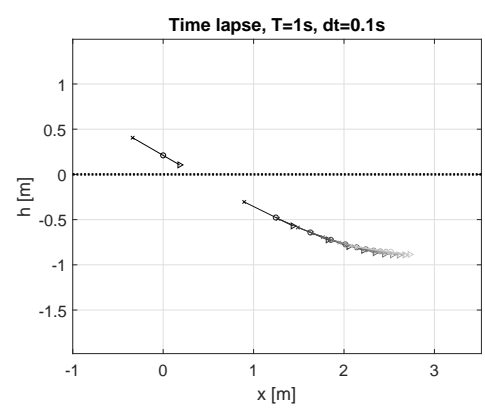

(c) Time lapse, $\theta_{i m p}=-30^{\circ}$

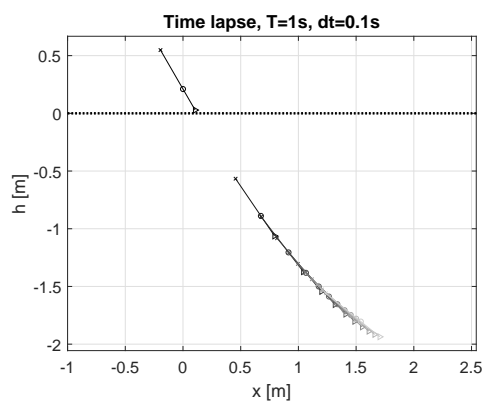

(d) Time lapse, $\theta_{i m p}=-60^{\circ}$
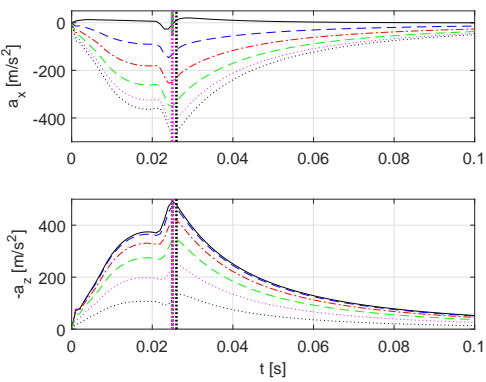

(b) Inertial-frame accelerations

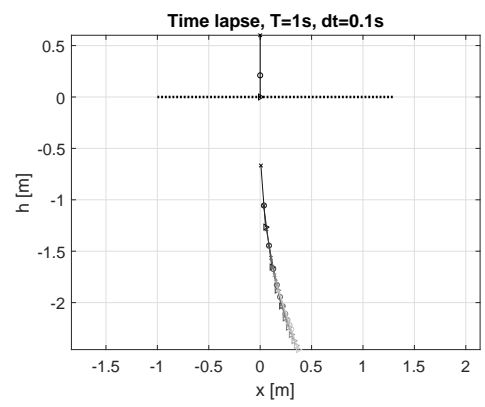

(e) Time lapse, $\theta_{i m p}=-90^{\circ}$

Figure 13. Water impact process for different entry angles $\theta_{i m p}(25 \mathrm{~m} / \mathrm{s}$ entry velocity); the dashed lines indicate the time at which the body is fully submerged; the bottom plots show time lapses over $1 \mathrm{~s}$. 

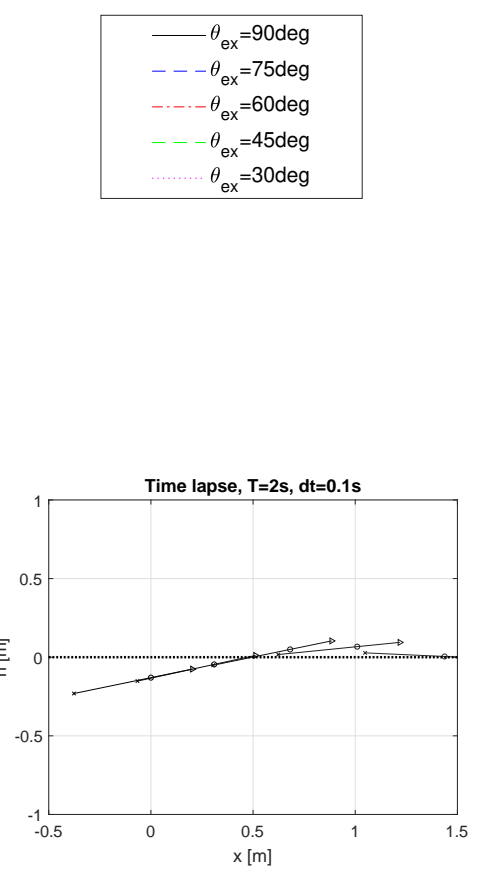

(c) $\theta_{\text {ex }}=15^{\circ}$
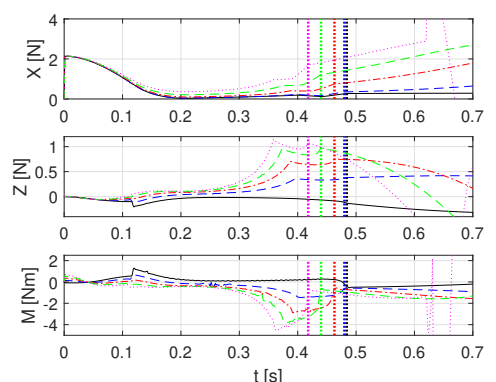

(a) Body-frame forces and pitch moment

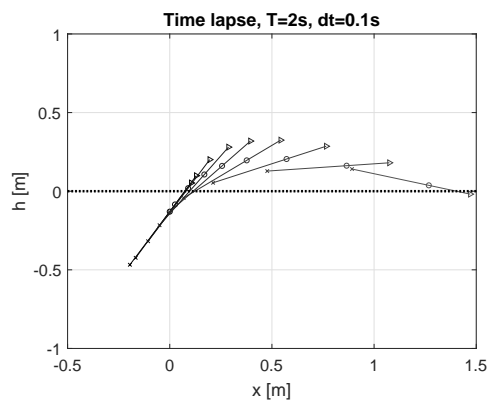

(d) $\theta_{e x}=60^{\circ}$
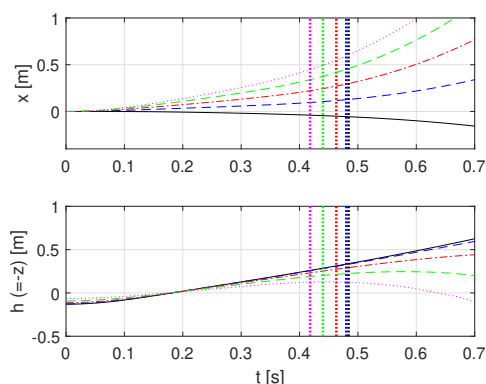

(b) Inertial-frame CG positions

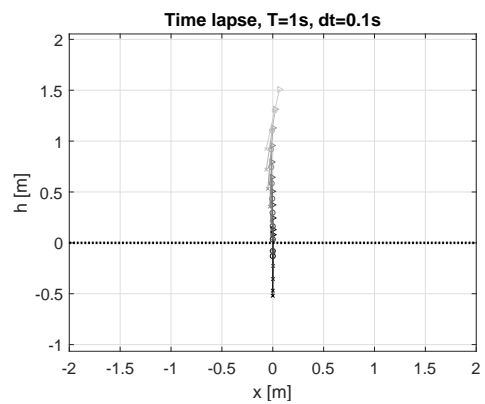

(e) $\theta_{e x}=90^{\circ}$

Figure 14. Water exit process for different exit angles $\theta_{e x}$, starting from near-floating conditions; the dashed lines indicate the time at which the body is fully out of the water (which for some conditions is never the case); the bottom plots show time lapses over $2 \mathrm{~s}$.

dynamics, and providing a detailed simulation framework supporting further control and navigation work on similar vehicles. The devised model includes aerial, underwater and transition phases of operation, and hence allows for all parts of the envisaged vehicle behaviour to be simulated. The aerodynamic forces were modelled using a system identification approach, based on wind tunnel data obtained for a similar robot, which led to an accurate low-order model accounting for the morphing wings of the vehicle. The remaining forces were characterised with simplified analytical models. Particular emphasis was placed on the air/water transitions, which were represented under full consideration of time-varying effects.

The resulting overall model was found to yield a detailed and plausible representation of the MAV, and retains a relatively simple structure, amenable for practical applications. The model was used to analyse the behaviour of the (type of) vehicle in different operation phases. The system dynamics were shown to vary considerably, due to both the wing-folding and the air-water transitioning, however the vehicle was found to be stable within the flight envelope considered and outside of the transition phases. A basic characterisation of the transition phases illustrated the forces occurring during water entry and exit and highlighted the difficulties of modelling and simulating these phases accurately, owing to the very large forces and short time scales. While a highly accurate representation may be unnecessary due to the short time scales, it is considered advisable to conduct an in-depth stability analysis of the time-varying components, and to consider modelling uncertainty when developing controllers for the transitions. This is particularly relevant for the more gradual water exit phase.

The model represents a useful basis for further simulation, analysis and control work, e.g. providing an initial testing possibility for an unconventional type of vehicle, and allowing for model-based control methods to be applied. Further work will focus on more advanced stability analysis and control of the transition phases. Experimental validation of the transition results would be a useful preliminary step. The final goal will be to implement and test the proposed system in real-life experiments, and to develop a control framework allowing for fully autonomous operation. 


\section{Acknowledgements}

This work was supported by EPSRC as part of the CASCADE programme grant (EP/R009953/1) and by the Royal Society Wolfson Fellowship (RSWF/R1/18000). The authors would additionally like to thank Alejandro Ortega, Raphael Zufferey and André Farinha for the helpful discussions.

\section{References}

${ }^{1}$ Wood, R., "The first takeoff of a biologically-inspired at-scale robotic insect," IEEE Transactions on Robotics, Vol. 24(2), 2008, pp. 341-347.

${ }^{2}$ Kovac, M., "Learning from nature how to land aerial robots," Science, Vol. 352, No. 6288, 2016, pp. 895-896.

${ }^{3}$ de Croon, G. C. H. E., Perçin, M., Remes, B. D. W., Ruijsink, R., and De Wagter, C., The DelFly Design, Aerodynamics, and Artificial Intelligence of a Flapping Wing Robot, Springer Netherlands, 2016, ISBN 978-94-017-9208-0.

${ }^{4}$ Baek, S. and Fearing, R., "Flight forces and altitude regulation of 12 gram i-bird," IEEE RAS and EMBS International Conference on Biomedical Robotics and Biomechatronics (BioRob), 2010.

${ }^{5}$ Siddall, R. and Kovač, M., "Launching the AquaMAV: Bioinspired design for aerial-aquatic robotic platforms," Bioinspiration and Biomimetics, Vol. 9, No. 3, 2014.

${ }^{6}$ Kovac, M., "The Bioinspiration Design Paradigm A Perspective for Soft Robotics," Soft Robotics, Vol. 1, No. 1, 2014.

${ }^{7}$ Ropert-Coudert Y, Gremillet, D., Ryan, P., Kato, A., Naito, Y., and Le Maho, Y., "Between air and water The plunge dive of the Cape gannet Morus capensis," International Journal of Avian Science, Vol. 146, 2004.

${ }^{8}$ Hedd, A., Regular, P. M., Montevecchi, W. A., Buren, A. D., Burke, C. M., and A., F. D., "Going deep Common murres dive into frigid water for aggregated, persistent and slow-moving capelin," Marine Biology, Vol. 156, No. 741, 2009.

${ }^{9}$ Yang, X., Wang, T., Liang, J., Yao, G., and Liu, M., "Survey on the novel hybrid aquatic-aerial amphibious aircraft aquatic unmanned aerial vehicle (AquaUAV)," Progress in Aerospace Sciences, 2015.

${ }^{10}$ Siddall, R. and Kovac, M., "Fast Aquatic Escape with a Jet Thruster," IEEE/ASME Transactions on Mechatronics, Vol. 22, No. 1, 2017, pp. 217-226.

${ }^{11}$ Siddall, R., Ancel, A., and Kovač, M., "Wind and water tunnel testing of a morphing aquatic micro air vehicle," Royal Society Interface Focus, Vol. 7, No. 1, 2017, pp. 20160085.

${ }^{12}$ Liang, J., Xing, Y., Tianmiao, W., Guocai, Y., and Zhao, W., "Design and Experiment of a Bionic Gannet for PlungeDiving," Journal of Bionic Engineering, Vol. 10, No. 3, 2013.

${ }^{13}$ Young, T. D., "Design and testing of an air-deployed unmanned underwater vehicle," AIAA Aviation Technology, Integration, and Operations Conf., 2014

${ }^{14}$ Alzu'bi, H., Mansour, I., and Rawashdeh, O., "Loon Copter Implementation of a hybrid unmanned aquatic-aerial quadcopter with active buoyancy control," Journal of Field Robotics, 2017.

${ }^{15}$ Stewart, W., Weisler, W., MacLeod, M., Powers, T., Defreitas, A., Gritter, A., Anderson, M., Peters, K., Gopalarathnam, A., and Bryant, M., "Design and demonstration of a seabird-inspired fixed-wing hybrid UAV-UUV system," Bioinspiration \& Biomimetics, 2018.

${ }^{16}$ Maia, M., Soni, P., and Diez-Garias, F., "Demonstration of an Aerial and Submersible Vehicle Capable of Flight and Underwater Navigation with Seamless Air-Water Transition," (arXiv:1507.01932), 2015.

${ }^{17}$ Chen, Y., Wang, H., Farrell Helbling, E., Jafferis, N. T., Zufferey, R., Ong, A., Ma, K., Gravish, N., Chirarattananon, P., Kovac, M., and Wood, R., "A biologically inspired, flapping-wing, hybrid aerial-aquatic microrobot," Science Robotics, 2017.

${ }^{18}$ Drews, P. J., Alves Neto, A., and M., C. M. F., "Hybrid unmanned aerial underwater vehicle Modeling and simulation," IEEE/RSJ Int. Conf. on Intelligent Robots and Systems, 2014.

${ }^{19}$ Tan, Y. H., Siddall, R., and Kovac, M., "Efficient Aerial-Aquatic Locomotion with a Single Propulsion System," IEEE Robotics $\&$ Automation Letters, Vol. 2, No. 3, 2017.

${ }^{20}$ de Visser, C. C., Mulder, J. A., and Chu, Q. P., "Global Nonlinear Aerodynamic Model Identification with Multivariate Splines," AIAA Atmospheric Flight Mechanics Conference, No. AIAA Paper 2009-5726, 2009.

${ }^{21}$ Nelson, R., Flight stability and automatic control, McGraw Hill, 1998.

${ }^{22}$ Yang, J., Feng, J., Li, Y., Liu, A., Hu, J., and Ma, Z., "Water-exit process modelling and added-mass calculation of the submarine-launched missile," Polish maritime research, Vol. 24, No. 95, 2017, pp. 152-164.

${ }^{23}$ Mishra, V. and Bhattacharya, S., "Translational Added Mass of Axisymmetric Underwater Vehicles with Forward Speed Using Computational Fluid Dynamics," Journal of Ship Research, Vol. 55, No. 3, 2011, pp. 185-195.

${ }^{24}$ Perrault, D., Bose, N., O'Young, S. D., and Williams, C. D., "Sensitivity of AUV added mass coefficients to variations in hull and control plane geometry," Ocean Engineering, Vol. 30, No. 5, 2003, pp. 645-671.

${ }^{25}$ Brennen, C. E., "A Review of added mass and fluid inertial forces," Tech. rep., Naval Civil Engineering Laboratory, 1982.

${ }^{26}$ Korotkin, A. I., Added Masses of Ship Structures, Vol. 1 of 88, Springer Netherlands, 1st ed., 2009.

${ }^{27}$ Wagner, H., "Über die Entstehung des dynamischen Auftriebes von Tragflügeln," Zeitschrift für Angewandte Mathematik und Mechanik, Vol. 5, 1925, pp. 17-35.

${ }^{28}$ Roe, S. M., Numerical and Experimental Analysis of Initial Water Impact of an Air-Dropped REMUS AUV, Master's thesis, Massachusets Institute of Technology, 2005.

${ }^{29}$ Shiffman, M. and Spencer, D. C., "The Force of Impact on a Cone Striking a Water Surface (Vertical Entry)," Communications on Pure and Applied Mathematics, 1951.

${ }^{30}$ Scolan, Y.-M. and Korobkin, A. A., "Water entry of a body which moves in more than six degrees of freedom," Proceedings of the Royal Society A, Vol. 471, 2015. 